\title{
¿Cómo mejorar la docencia en Electrónica de Dispositivos? Guía de actividades
}

\author{
JUAN ANTONIO LEÑERO BARDALLO \\ Departamento de Electrónica y Electromagnetismo \\ Universidad de Sevilla \\ jlenero@us.es \\ ORCID: https://orcid.org/0000-0002-1741-9743 \\ D.O.I.: http://dx.doi.org/10.12795/JDU.2018.i01.20 \\ Pp.: 356-373
}

\section{Resumen}

La implementación de un Ciclo de Mejora Docente (CDM) se ha realizado en la asignatura 'Electrónica Física'. La asignatura es el primer contacto de los estudiantes con el estudio de la física de los dispositivos semiconductores, siendo muchos de los conceptos impartidos totalmente novedosos para el alumnado. El índice de aprobados de la asignatura ha sido tradicionalmente bajo, aun cuando la nota de corte de algunas de las titulaciones de los alumnos que la cursan es muy alta. El ciclo de mejora se apoya en la elaboración de guía de actividades y boletines de problemas que se adaptan a las necesidades del alumnado, actuando el profesor como guía en la resolución de los problemas planteados.

Palabras claves: Electrónica física, Grado en Física, Grado en Ingeniería de Materiales, Doble grado en Física y Matemáticas, Doble grado 
en Física e Ingeniería de Materiales; docencia universitaria, experimentación docente universitaria, guía de actividades y boletines de problemas.

\section{Breve descripción del contexto de la intervención}

Se ha propuesto una experiencia de innovación docente para la asignatura Electrónica Física. Se trata de una asignatura troncal y obligatoria de seis créditos, que se imparte en el segundo cuatrimestre de forma simultánea en varias titulaciones: en el Grado en Física, en el Grado en Ingeniería de Materiales y en los dobles grados en Física y Matemáticas y en Física e Ingeniería de Materiales.

Es la primera asignatura relacionada con la Electrónica que se imparte en todas estas titulaciones y que requiere un esfuerzo extra por parte del alumnado que la cursa por primera vez.

La carga docente de la asignatura ha pasado de nueve a seis créditos, sin que el temario que se imparte, se haya reducido de forma acorde.

De todo lo anterior se extrae que el indice de aprobados sea relativamente bajo (por debajo del 60\% en primera convocatoria), en comparación con otras asignaturas de la titulación. Este resultado preocupa toda vez que los alumnos acceden a las titulaciones con notas de corte muy altas. En este contexto se produce la necesidad de realizar el ciclo de mejora docente (CMD), analizando el estado del alumnado, proponiendo medidas de mejora, evaluando el resultado de la experiencia docente realizada, para concluir con la propuesta de nuevas mejoras para los próximos cursos.

La docencia se divide en clases de teoría (tres horas semanales) y en clases de problemas (una hora semanal). Las actividades del ciclo de mejora se han desarrollado en los seminarios prácticos dedicados a la realización de problemas. 


\section{Descripción de las clases habituales y evolución de las mismas durante el curso académico 2016-2017}

La asignatura es fuertemente teórica, siendo las clases de teoría el pilar de la asignatura. Las clases (seminarios) de problemas son extensiones de las clases teóricas donde se analizan casos particulares, en mayor detalle que en las clases teóricas. En algunos casos, se resuelven problemas que permiten hacer un análisis cuantitativo del comportamiento de los dispositivos semiconductores y los efectos de segundo orden que los afectan.

Las clases de teoría son de tipo magistral en las que se usan diapositivas para introducir los conceptos de la asignatura. La pizarra se utiliza como herramienta para realizar demostraciones matemáticas e ilustraciones de los conceptos que se van introduciendo en clase.

Los seminarios de problemas son también de tipo magistral en los que el profesor resuelve problemas de boletines, que se encuentran previamente disponibles en la Plataforma de Enseñanza Virtual. Los alumnos tienen la opción de intentar resolverlos en casa y comprobar en clase si su solución es la misma que la propuesta por el profesor. Para ello cuentan con el material impartido en las clases de teoría. En la práctica, los alumnos esperan a las clases de problemas, para ver la solución aportada por el profesor a los problemas.

\section{Tabla 1}

Esquema de los seminarios de problemas antes de la puesta en marcha del CMD

\begin{tabular}{|l|l|}
\hline \multicolumn{2}{|l|}{ Estructura de los seminarios previa al Ciclo de Mejora Docente } \\
\hline 1 & Publicación de actividades en la Plataforma de Enseñanza Virtual \\
\hline 2 & $\begin{array}{l}\text { Preparación de la pizarra, presentación, e introducción de la mate- } \\
\text { ria a tratar }\end{array}$ \\
\hline 3 & Lectura y explicación de los problemas que se van a resolver en clase \\
\hline
\end{tabular}

Jornadas de Formación e Innovación Docente del Profesorado | № 1 (2018) Esta obra se distribuye con la licencia Creative Commons 


\begin{tabular}{|l|l|}
\hline 4 & Resolución de los problemas en la pizarra \\
\hline 5 & $\begin{array}{l}\text { Análisis de los resultados. Discusión de los mismos. Se establecen } \\
\text { vínculos con magnitudes y propiedades de la materia conocidas por } \\
\text { el alumnado }\end{array}$ \\
\hline 6 & $\begin{array}{l}\text { Resumen del problema resuelto. Justificación y encuadre en la } \\
\text { asignatura }\end{array}$ \\
\hline 7 & $\begin{array}{l}\text { Tiempo dedicado a que los estudiantes desarrollen la resolución del } \\
\text { problema planteado en clase }\end{array}$ \\
\hline
\end{tabular}

Tabla 2

Secuencia habitual antes de implementar el CMD

Planteamiento del problema + Resolución en la pizarra + Análisis de resultados y discusión + Justificación de la tarea realizada

\section{Análisis de las clases durante el curso académico 2016-2017}

Durante el desarrollo de las clases ya impartidas a comienzo del presente curso, observé la necesidad de realizar un cambio en la dinámica de la clase debido a las siguientes observaciones:

1. Era necesario que los alumnos intentasen realizar los problemas propuestos antes de los seminarios

2. Muchos estudiantes se limitaban a copiar las soluciones propuestas por el profesor, sin establecer ningún tipo de razonamiento crítico

3. Las intervenciones de los alumnos en clase no eran frecuentes y las dudas que plantean son muy básicas, en la mayoría de los casos

4. De la mera observación del desarrollo de la clase resultaba difícil hacer una valoración profunda de las carencias del alumnado

5. Era necesario establecer medidas para corregir la tendencia actual

Jornadas de Formación e Innovación Docente del Profesorado I № 1 (2018) Esta obra se distribuye con la licencia Creative Commons 


\section{Modelo metodológico habitual antes de realizar el ciclo de mejora}

Actividades de iniciación: Planteamiento del problema Actividades de profundización: Resolución en la pizarra Actividades de seguimiento: Análisis de resultados y discusión

Actividades de cierre: Justificación de la tarea realizada

\section{Modelo metodológico ideal que se pretendía conseguir}

El modelo metodológico ideal se ha dirigido hacia la consecución de los objetivos buscados, Porlan (2017), quedando destacados los siguientes puntos clave:

1. Comenzar con la realización de preguntas clave para conocer el grado y el nivel de conocimiento de los estudiantes Bain (2007)

2. Evaluar las respuestas de los estudiantes a las preguntas clave

3. Plantear una secuencia de problemas dirigidos y ajustados a los niveles de respuesta dados por los estudiantes en relación a las preguntas clave realizadas al inicio del ciclo de mejora.

4. Los estudiantes desarrollan y resuelven los problemas planteados, debatiendo entre ellos y con el profesor las dificultades que se encuentran durante el ejercicio de resolución de problemas.

5. El profesor debate con los estudiantes las dificultades obtenidas y realiza resumen de los aspectos destacados de la secuencia de problemas

\section{Tabla 3}

Secuencia del modelo metodológico ideal del CMD

Preguntas clave + Respuestas a preguntas clave + Problemas dirigidos + Trabajo autónomo de los alumnos + Debate final 


\section{Diseño del Ciclo de Mejora Docente}

El diseño se realiza en base al modelo metodológico ideal anteriormente descrito.

Mapa de contenidos y preguntas clave asociadas

El mapa de contenidos a impartir en la asignatura se elaboró teniendo en cuenta los tres bloques de contenidos básicos para la docencia en Electrónica de Dispositivos y las preguntas clave asociadas a los contenidos básicos.

El mapa de contenidos permite situar la estructura y las relaciones que se producen entre contenidos que debe alcanzar en su aprendizaje el estudiante.

En el mapa de contenidos se ha asociado a cada contenido una o varias preguntas clave, identificadas con la letra "p" y un número " $n$ " estableciendo el código "pn".

En la tabla 4 de la página siguiente quedan identificadas las relaciones del mapa de contenidos.

A continuación de la tabla 4 se indican las preguntas del cuestionario identificadas con el código "pn" expresadas en el mapa.

Jornadas de Formación e Innovación Docente del Profesorado | № 1 (2018) Esta obra se distribuye con la licencia Creative Commons 
Tabla 4

Mapa de contenidos del CMD

\begin{tabular}{|c|c|c|c|c|c|}
\hline \multirow{3}{*}{$\begin{array}{l}\text { Carga } \\
(\mathrm{p} 1, \mathrm{p} 4)\end{array}$} & & CONTENID & OS & \multirow{4}{*}{$\begin{array}{l}\text { Teorema de } \\
\text { Gauss } \\
(\mathrm{p} 2)\end{array}$} & \multirow{3}{*}{$\begin{array}{l}\text { SESIONES } \\
\text { Dos } \\
\text { sesiones }\end{array}$} \\
\hline & 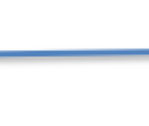 & $\begin{array}{l}\text { Campo } \\
\text { eléctrico } \\
\text { (p1 y p3) }\end{array}$ & & & \\
\hline & $\begin{array}{l}\text { Cargas } \\
\text { puntuales } \\
\text { (p3) }\end{array}$ & $\begin{array}{l}\text { Densidades } \\
\text { Volumétricas } \\
\text { (p3) }\end{array}$ & $\begin{array}{l}\text { Perfiles } \\
\text { arbitrarios } \\
\text { de carga } \\
\text { (p3) }\end{array}$ & & \\
\hline \multirow[t]{3}{*}{$\begin{array}{l}\text { Carga } \\
(\mathrm{p} 1, \mathrm{p} 4)\end{array}$} & $\begin{array}{l}\text { Campo } \\
\text { eléctrico } \\
(\mathrm{p} 1, \mathrm{p} 3)\end{array}$ & $\begin{array}{l}\text { Potencial } \\
\text { electroestático } \\
\text { (p4) }\end{array}$ & & & \\
\hline & & $\begin{array}{l}\text { Cálculo de } \\
\text { potencial (p6) }\end{array}$ & & & \\
\hline & $\begin{array}{l}\text { Cargas } \\
\text { puntuales } \\
\text { (p6) }\end{array}$ & $\begin{array}{l}\text { Densidades } \\
\text { volumétricas } \\
\text { de carga } \\
\text { (p6) }\end{array}$ & $\begin{array}{l}\text { Perfiles } \\
\text { arbitrarios } \\
\text { de carga } \\
\text { (p6) }\end{array}$ & (p5) & sesiones \\
\hline \multirow[t]{2}{*}{$\begin{array}{l}\text { Primera Ley } \\
\text { (p8) }\end{array}$} & $\begin{array}{l}\text { Segunda } \\
\text { Ley } \\
\text { (p9) }\end{array}$ & $\begin{array}{l}\text { Teoría de } \\
\text { Circuitos } \\
\text { (p10) }\end{array}$ & & $\begin{array}{l}\text { Leyes de } \\
\text { Kirchhoff }\end{array}$ & $\begin{array}{l}\text { Dos } \\
\text { sesiones }\end{array}$ \\
\hline & & $\begin{array}{l}\text { Nudos } \\
\text { (p11) }\end{array}$ & $\begin{array}{l}\text { Mallas } \\
\text { (p11) }\end{array}$ & & \\
\hline $\begin{array}{l}\text { Movimiento } \\
\text { de cargas } \\
(\mathrm{p} 12)\end{array}$ & & $\begin{array}{l}\text { Corrientes de } \\
\text { arrastre } \\
\text { (p13) }\end{array}$ & $\begin{array}{l}\text { Flujos } \\
\text { difusivos } \\
\text { (p13) }\end{array}$ & $\begin{array}{l}\text { Ecuaciones } \\
\text { de } \\
\text { Transporte } \\
\text { (p12,p13) }\end{array}$ & $\begin{array}{l}\text { Una } \\
\text { sesión }\end{array}$ \\
\hline
\end{tabular}

Jornadas de Formación e Innovación Docente del Profesorado | № 1 (2018)

(C) (i) $(2) \bigcirc$ Esta obra se distribuye con la licencia Creative Commons

Internacional (CC BY-NC-ND 4.0.) 
Relación de preguntas del cuestionario inicial que se entrega al comienzo de la primera sesión del ciclo de mejora

(p1). Qué relación existe entre la carga y el campo eléctrico?

(p2). ¿Qué establece el Teorema de Gauss?

(p3). ¿Qué sucede en un campo eléctrico cuando hay cargas puntuales, hay densidades volumétricas o hay perfiles arbitrarios de carga?

(p4). ¿Qué relación hay entre el potencial electroestático y la carga? ¿y entre el potencial electrostático y el campo eléctrico?

(p5). ¿Qué establece el Teorema de Poisson?

(p6). ¿Cómo de obtiene el cálculo de potencial generado por cargas puntuales, densidades volumétricas de carga y perfiles arbitrarios de carga?

(p7). ¿Cuándo y cómo uso las Leyes de Kirchhoff?

(p8). ¿Qué identifica la Primera Ley?

(p9). ¿En qué puedo aplicar la Segunda Ley?

(p10). ¿Cómo aplico las Leyes de Kirchhoff para resolver circuitos?

(p11). ¿Cómo puedo aplicar el análisis de nudos y mallas?

(p12). ¿Qué mecanismos gobiernan el movimiento de cargas?

(p13). ¿En qué podemos aplicar los cálculos básicos de las corrientes de arrastre y los flujos difusivos?

\section{Modelo metodológico posible y secuencia de actividades programadas}

El modelo metodológico posible se ajusta a las limitaciones reales asociadas al tiempo disponible y a la capacidad real de los alumnos, incorporando como apoyo a las actividades docentes una guía de actividades y boletines 
de problemas encaminados a conducir a los estudiantes en su proceso de enseñanza-aprendizaje en la asignatura.

\section{Tabla 5}

Distribución de actividades del modelo metodológico posible (sesión estándar 60 minutos)

\begin{tabular}{|l|l|}
\hline 10 minutos & Realización de preguntas clave \\
\hline 5 minutos & $\begin{array}{l}\text { Evaluación de las respuestas de los estudiantes a las pre- } \\
\text { guntas clave mediante la realización de encuesta debate }\end{array}$ \\
\hline 5 minutos & $\begin{array}{l}\text { Propuesta de secuencia de problemas de la guía de } \\
\text { actividades }\end{array}$ \\
\hline 10 minutos & Inicio de la resolución de problemas por los estudiantes \\
\hline 5 minutos & $\begin{array}{l}\text { Debate sobre dificultades encontradas por los estudiantes } \\
\text { en el encerado. Los alumnos explican los problemas que } \\
\text { han tenido. }\end{array}$ \\
\hline 10 minutos & $\begin{array}{l}\text { El profesor esquematiza la posible solución del problema } \\
\text { material elaborado la resolución del problema }\end{array}$ \\
\hline 15 minutos & $\begin{array}{l}\text { El profesor soluciona el problema. Actividades de cierre. } \\
\text { Debate y conclusiones obtenidas }\end{array}$ \\
\hline
\end{tabular}

El modelo posible incorpora, nuevos objetivos docentes dirigidos a:

\section{- El aprendizaje autónomo de los estudiantes}

- El conocimiento del nivel de los estudiantes y sus carencias

- Una revisión profunda de los materiales docentes disponibles

La duración total del ciclo de mejora fue de 7 horas, estructuradas en 7 sesiones de una hora de duración cada una de ellas. El ciclo de mejora docente se ha realizado en dos grupos distintos, siendo la duración total de la innovación docente de $\mathbf{7} \times \mathbf{2}=\mathbf{1 4}$ horas.

Se detallan a continuación la secuencia de actividades que se realizó en cada sesión. Se utilizaron los siguientes códigos para cada tipo de actividad:

C: Cuestionario 
T: Tarea a realizar por el estudiante

A: Acciones durante el desarrollo de la actividad / tarea a realizar

CR: Actividades de cierre

En la tabla 6 se incorpora como ejemplo de actividades, lo realizado en la sesión 2 del CMD

\section{Tabla 6}

Ejemplo de actividades. Sesión 2 del CMD

\begin{tabular}{|l|l|l|l|}
\hline \multicolumn{2}{|l|}{ Sesión 2: 19 de abril. El Teorema de Gauss. } \\
\hline $\begin{array}{l}\text { Sinopsis: La sesión se centra en el repaso y en la resolución de casos prácti- } \\
\text { cos de interés para la asignatura, en los que haya que hacer uso del Teorema de } \\
\text { Gauss. El alumno debe saber relacionar la existencia de carga en el espacio con } \\
\text { la existencia de un campo eléctrico }\end{array}$ \\
\hline $\begin{array}{l}\text { No de } \\
\text { actividad }\end{array}$ & C & $\begin{array}{l}\text { iQué sucede en un campo eléctrico cuando hay cargas puntua- } \\
\text { les, hay densidades volumétricas o hay perfiles arbitrarios de } \\
\text { carga? }\end{array}$ \\
\hline 1 & T & $\begin{array}{l}\text { Problema tipo II del cálculo del campo eléctrico, mediante el } \\
\text { Teorema de Gauss. Se propone hacer el cálculo en las siguien- } \\
\text { tes situaciones: } \\
\text { 1. Con cargas puntuales } \\
\text { 2. Distribución uniforme de carga por unidad de volumen } \\
\text { 3. Volumen con una distribución de carga arbitraria }\end{array}$ \\
\hline 3 & CR & $\begin{array}{l}\text { Realización de preguntas clave asociadas a los conteni- } \\
\text { dos de la sesión. } \\
\text { Evaluación de las respuestas de los estudiantes a las pre- } \\
\text { guntas clave mediante la realización de encuesta debate. } \\
\text { Inicio de la resolución de problemas por los estudiantes } \\
\text { Debate sobre dificultades encontradas por los estudian- } \\
\text { tes en el encerado } \\
\text { El profesor esboza una posible estrategia para abordar la } \\
\text { resolución del problema } \\
\text { Los estudiantes buscan por sus medios y con ayuda del } \\
\text { material elaborado la resolución del problema }\end{array}$ \\
\hline $\begin{array}{l}\text { El profesor esquematiza la posible solución del problema. } \\
\text { Actividades de cierre. Debate y conclusiones obtenidas }\end{array}$ \\
\hline 1
\end{tabular}


La primera y la última sesión incluyeron además un cuestionario inicial/final de 30 minutos para la evaluación del ciclo de mejora que se detalla a continuación.

\section{Cuestionario inicial-final para evaluar la evolución de los estudiantes}

Con la intención de evaluar la experiencia docente y la evolución de los estudiantes, se elaboró un cuestionario que se pasó a los estudiantes en la primera y la última sesión del ciclo de mejora. La tabla siguiente refleja las cuestiones que se plantearon.

\section{Tabla 7}

\section{Cuestionario inicial/final}

\begin{tabular}{|c|c|c|}
\hline \multicolumn{3}{|c|}{ Cuestionario Inicial/Final del Ciclo de Mejora } \\
\hline Número & Pregunta & Descripción \\
\hline 1 & $\begin{array}{l}\text { ¿Cuál es la relación entre la dis- } \\
\text { tribución volumétrica de carga y } \\
\text { el campo eléctrico? }\end{array}$ & $\begin{array}{l}\text { Pregunta encaminada a que el } \\
\text { alumno formule el Teorema de } \\
\text { Gauss }\end{array}$ \\
\hline 2 & $\begin{array}{l}\text { Problema práctico: Dada una dis- } \\
\text { tribución de carga en las con- } \\
\text { diciones que se indican en el } \\
\text { enunciado, se pide al alumno } \\
\text { que calcule el campo eléctrico }\end{array}$ & $\begin{array}{l}\text { Problema de cálculo en que el } \\
\text { alumno debe utilizar el Teo- } \\
\text { rema de Gauss para resolverlo. }\end{array}$ \\
\hline 3 & $\begin{array}{l}\text { ¿Cuál es la relación entre el po- } \\
\text { tencial eléctrico y la distribución } \\
\text { volumétrica de carga? }\end{array}$ & $\begin{array}{l}\text { Pregunta encaminada a que el } \\
\text { alumno formule el Teorema de } \\
\text { Poisson }\end{array}$ \\
\hline 4 & $\begin{array}{l}\text { Problema práctico: Dada una dis- } \\
\text { tribución de carga en las con- } \\
\text { diciones que se indican en el } \\
\text { enunciado, se pide al alumno } \\
\text { que calcule el potencial eléctrico }\end{array}$ & $\begin{array}{l}\text { Problema de cálculo en que } \\
\text { el alumno debe utilizar el } \\
\text { Teorema de Poisson para } \\
\text { resolverlo. }\end{array}$ \\
\hline 5 & $\begin{array}{l}\text { Enuncie los mecanismos que } \\
\text { pueden hacer que cargas (elec- } \\
\text { trones y huecos) se desplacen en } \\
\text { el espacio }\end{array}$ & $\begin{array}{l}\text { Pregunta encaminada a que el } \\
\text { alumno enuncie los dos meca- } \\
\text { nismos que gobiernan el des- } \\
\text { plazamiento espacial de cargas: } \\
\text { el arrastre y la difusión. }\end{array}$ \\
\hline
\end{tabular}




\begin{tabular}{|l|l|l|}
\hline 6 & $\begin{array}{l}\text { Problema práctico: Estudio del } \\
\text { desplazamiento de cargas debido } \\
\text { a fenómenos de arrastre, a fenó- } \\
\text { menos de difusión y fenómenos } \\
\text { de arrastre y difusión. }\end{array}$ & $\begin{array}{l}\text { Problema de cálculo del des- } \\
\text { plazamiento que experimentan } \\
\text { cargas debido a la presencia } \\
\text { de un campo eléctrico (flujo de } \\
\text { arrastre), a la presencia de un } \\
\text { gradiente en la distribución vo- } \\
\text { lumétrica de carga (flujo difu- } \\
\text { sivo) y debido a la presencia } \\
\text { conjunta de ambos fenómenos. }\end{array}$ \\
\hline $\begin{array}{l}\text { En el cuestionario final, se incluyeron preguntas adicionales para valorar el } \\
\text { CMD: }\end{array}$ & $\begin{array}{l}\text { Valore el ciclo de mejora de forma global. ¿Piensa que le ha sido } \\
\text { útil? }\end{array}$ \\
\hline F.1 & $\begin{array}{l}\text { Comente cuál de las sesiones le ha resultado más provechosa y } \\
\text { por qué }\end{array}$ \\
\hline F.2 & $\begin{array}{l}\text { Indique posibles mejoras futuras que se le ocurran para el ciclo de } \\
\text { mejora }\end{array}$ \\
\hline
\end{tabular}

\section{Aplicación del Ciclo de Mejora Docente}

\section{Relato Resumido de las Sesiones}

Se resumen a continuación el desarrollo de las siete sesiones del ciclo de mejora en los dos grupos de clase en las que se llevaron a cabo.

\section{Tabla 8}

Resumen sesiones

\begin{tabular}{|l|l|}
\hline Sesión & Resumen \\
\hline 1 & $\begin{array}{l}\text { La primera sesión se desarrolló según el esquema previsto. Inicial- } \\
\text { mente el alumnado se mostró sorprendido por las cuestiones del } \\
\text { cuestionario inicial, puesto que hacian referencia a conceptos ya es- } \\
\text { tudiados por ellos en otras asignaturas. Muchos no entendieron el } \\
\text { posible vínculo de esas cuestiones con la asignatura. Las actividades } \\
\text { de cierre se realizaron más rápido de lo previsto. }\end{array}$ \\
\hline
\end{tabular}

Jornadas de Formación e Innovación Docente del Profesorado | № 1 (2018) Esta obra se distribuye con la licencia Creative Commons 


\begin{tabular}{|l|l|}
\hline 2 & $\begin{array}{l}\text { En la segunda sesión, el alumnado encontró dificultades para resol- } \\
\text { ver las actividades propuestas. Los casos prácticos que tuvieron que } \\
\text { resolver eran de menor dificultad a los que han estudiado previa- } \\
\text { mente en otras asignaturas de Electromagnetismo. Una mejor coor- } \\
\text { dinación con los profesores de otras asignaturas relacionadas, puede } \\
\text { ayudar a solventar esta dificultad. }\end{array}$ \\
\hline 3 & $\begin{array}{l}\text { La sesión se desarrolló según el esquema previsto; los alumnos aca- } \\
\text { baron las actividades propuestas en el tiempo estimado. En futuras } \\
\text { sesiones deberín introducirse actividades adicionales, para un me- } \\
\text { jor aprovechamiento de la sesión. }\end{array}$ \\
\hline 4 & $\begin{array}{l}\text { Los conceptos vistos en esta sesión eran de una dificultad conceptual } \\
\text { mayor que los de anteriores sesiones. Ello hizo que algunos alumnos } \\
\text { se desmotivaran y desistieran. Un enfoque gradual proponiendo más } \\
\text { actividades relacionadas en la sesión anterior puede ayudar a asimi- } \\
\text { lar los contenidos a tratar en la sesión. }\end{array}$ \\
\hline 5 & $\begin{array}{l}\text { Los contenidos de esta sesión eran conocidos por los alumnos. Sin } \\
\text { embargo, se abordaban desde un punto de vista más simplificado y } \\
\text { práctico que en otras asignaturas de cursos anteriores. Algunos alum- } \\
\text { nos perdieron mucho tiempo debatiendo qué estrategia seguir para } \\
\text { resolver las actividades planteadas. }\end{array}$ \\
\hline 6 & $\begin{array}{l}\text { Esta clase sesión se utilizó para resolver circuitos electrónicos ya es- } \\
\text { tudiados en la asignatura, utilizando las Leyes de Kirchhoff. Al prin- } \\
\text { cipio, los alumnos con mucha dificultad trataron de identificar los } \\
\text { nuevos elementos que aparecian en los esquemas eléctricos que se } \\
\text { les proporcionó. Poco a poco, fueron vinculando sus conocimientos } \\
\text { de análisis de circuitos previos con los nuevos dispositivos semicon- } \\
\text { ductores utilizados en la asignatura. }\end{array}$ \\
\hline $\begin{array}{l}\text { Esta sesión se desarrolló según lo previsto. Sin embargo, las activida- } \\
\text { des propuestas a los alumnos resultaron ser demasiado extensas, ya } \\
\text { que en esta sesión se realizó de nuevo el cuestionario inicial y el de- } \\
\text { bate final de la asignatura. Para sesiones futuras, se propone dedicar } \\
\text { una sesión en exclusiva para aplicar las Ecuaciones del Transporte. } \\
\text { En el debate final, los alumnos dieron su opinión sobre el cmD. }\end{array}$ \\
\hline 5
\end{tabular}

\section{Evaluación del aprendizaje de los estudiantes}

Se utilizaron tres métricas distintas: la comparativa de los resultados de los cuestionarios inicial/final, los resultados obtenidos en el examen y las propias opiniones de los estudiantes sobre la experiencia docente. Se analizan a continuación cada uno de ellos por separado. 


\section{Resultados del cuestionario inicial/final}

Para cada una de las preguntas y/o ejercicios de los cuestionarios inicial/final se indican los resultados en la primera vez y en la segunda vez que realizaron el cuestionario.

\section{Tabla 9}

Extracto de resultados de cuestionario inicial y final

\begin{tabular}{|c|c|c|c|c|}
\hline & $\begin{array}{c}\% \text { no } \\
\text { responden }\end{array}$ & $\begin{array}{c}\% \text { respuesta } \\
\text { errónea }\end{array}$ & $\begin{array}{c}\% \text { respuesta } \\
\text { satisfactoria }\end{array}$ & $\begin{array}{c}\% \text { respuesta } \\
\text { excelente }\end{array}$ \\
\hline $\begin{array}{c}\text { Número de } \\
\text { pregunta }\end{array}$ & Cuestionario & Cuestionario & Cuestionario & Cuestionario \\
\cline { 2 - 5 } & Inicial/Final & Inicial/Final & Inicial/Final & Inicial/Final \\
\hline 1 & $5 / 4$ & $62 / 29$ & $28 / 60$ & $5 / 7$ \\
\hline 2 & $6 / 4$ & $50 / 24$ & $40 / 64$ & $4 / 8$ \\
\hline 3 & $4 / 2$ & $47 / 20$ & $51 / 70$ & $0 / 8$ \\
\hline 4 & $5 / 3$ & $61 / 39$ & $33 / 55$ & $1 / 3$ \\
\hline 5 & $7 / 3$ & $60 / 37$ & $32 / 50$ & $1 / 10$ \\
\hline 6 & $4 / 1$ & $50 / 35$ & $46 / 55$ & $0 / 9$ \\
\hline
\end{tabular}

\section{Resultados preliminares de los exámenes de la asignatura}

A fecha de redacción de esta comunicación solo se ha realizado el primer examen parcial de la asignatura. Los resultados de este examen no son concluyentes porque muchos alumnos optan por no realizar el examen parcial y examinarse de la asignatura completa en las convocatorias de junio y sucesivas. 
Análisis de resultados del primer examen parcial de este curso respecto al mismo examen parcial del curso anterior.

Los errores de cálculo obtenidos al aplicar el Teorema de Gauss y el Teorema de Poisson se redujeron

Los resultados en el problema de análisis de circuitos propuesto mejoraron. Quizás el hecho de forzar a los alumnos a tratar de resolver por sí mismos un circuito con componentes electrónicos hizo que pudieran comprobar la dificultad asociada

Los estudiantes, en su mayoría, no necesitaron tiempo adicional para responder a las cuestiones planteadas. En la asignatura, tradicionalmente, los exámenes han durado más de lo estipulado, siendo habitual que se dé un tiempo de gracia para responder

\section{Opiniones de los estudiantes en la encuesta realizada}

Junto al cuestionario final, se pidió a los estudiantes que respondieran a una encuesta sobre el ciclo de mejora, destacando aquellos aspectos del CMD que consideraban mejorable.

\section{Tabla 11}

Aspectos destacados de la encuesta realizada por los estudiantes respecto al CMD

Duración del ciclo de mejora: Los alumnos destacaron que la duración (siete sesiones) fue insuficiente

Intensidad de las sesiones: Los alumnos manifestaron que las tareas propuestas para algunas sesiones eran demasiadas, para el tiempo disponible. Especialmente, la última sesión, en la que además de realizar tareas, se realizó el cuestionario final y se pasó una encuesta

Los temas seleccionados para las sesiones del CMD no cubrían todo el temario de la asignatura. Los alumnos mostraron opiniones dispares sobre los temas que podrían incluirse o añadirse en ediciones futuras 


\section{Evaluación del Ciclo de Mejora Docente}

\section{Cuestiones a mantener y cambios a introducir}

Se mantienen la estructura de las sesiones y su duración. Los resultados obtenidos en el ciclo de mejora y las opiniones de los alumnos justifican una ampliación de la duración de las sesiones del mismo. En base a las escaleras de aprendizaje de los alumnos, el impacto del CMD se considera positivo. Se propone que en ediciones futuras el CDM comience junto con las clases.

El tiempo dedicado a las Ecuaciones del Transporte resultó ser insuficiente, dada la complejidad y la novedad de las mismas. Se propone pasar de una a dos sesiones de trabajo dedicadas al estudio de las ecuaciones en ediciones futuras. El rendimiento del alumnado indica que es necesario dedicar más tiempo al análisis de circuitos que al repaso de la Ley de Gauss. Se propone hacer una redistribución temporal asociada a cada uno de estos temas, acorde con los resultados obtenidos. Se estima necesario que en la primera y en la última sesión del CMD se amplíe el tiempo destinado a realizar los cuestionarios iniciales/finales, alcanzando gran parte del desarrollo de ambas sesiones.

\section{Aspectos que se pretenden incorporar a la práctica docente habitual.}

Se ha comprobado que los alumnos entienden el grado de complejidad de la asignatura y la necesidad de llevarla al día, al forzarles a resolver ejercicios en clase. El debate generado ha sido positivo, puesto que los alumnos han establecido vínculos entre ellos, que ha permitido un aprendizaje colaborativo. Ha sido habitual que varios alumnos hayan formado grupos de estudio para resolver 
problemas de exámenes de cursos pasados, tras la experiencia iniciada en las clases del CMD. El hecho de que el material didáctico estuviera disponible por adelantado ha sido valorado positivamente por el alumnado. El cuestionario final sobre la experiencia docente ha sido útil como herramienta de evaluación.

\title{
Principios didácticos argumentados que han guiado la experiencia docente y que deben permanecer en el futuro
}

\author{
La tabla 12 resume los principios
}

\section{Tabla 12}

Principios didácticos que han fundamentado la innovación docente en el proceso de enseñanza-aprendizaje y que deben permanecer en la asignatura.

\begin{abstract}
El aprendizaje basado en un trabajo previo, continuado en el tiempo, es más efectivo y perdura más que un aprendizaje a ráfagas, basado en estudio intenso antes del examen Finkel (2008)

La adopción de un enfoque constructivista Delval (1997), según el cual el alumno es el verdadero protagonista de su aprendizaje, teniendo éste un papel activo y, además, evalúa continuamente y somete a crítica sus propios conocimientos Gómez Rijo (2010)
\end{abstract}

La creación de un entorno de trabajo y de un entorno enriquecedor que lleve al alumno a trabajar de forma continuada y autónoma, y a plantearse interrogantes sobre los conceptos impartidos, y a aprender por sí mismos Bain (2007)

La elección de un tema de trabajo de interés por los estudiantes y que esté relacionado con los dispositivos electrónicos que usan a diario, éste hecho tradicionalmente ha dado buenos resultados pedagógicos Ausubel (1978) 


\section{Conclusiones}

Se ha propuesto el uso de una herramienta docente para identificar aquellos aspectos de la asignatura 'Electrónica Física' que generan mayor dificultad en el proceso de aprendizaje. La experiencia ha permitido tanto al alumnado como al profesorado identificar los contenidos de la asignatura que deben abordarse en mayor profundidad y en cada momento. En base a ello, los alumnos han comenzado a resolver problemas con mayor prontitud que en cursos anteriores. Se propone la aplicación del modelo en los próximos cursos. El CMD ha supuesto un cambio en la dinámica de la clase, con respecto a cursos anteriores.

\section{Bibliografía}

Porlan, R et al. (2017). Enseñanza Universitaria. Cómo mejorarla (2a edición). Morata.

Finkel, D. (2008). Dar clase con la boca cerrada. Valencia: Publicaciones de la Universidad de Valencia.

Ausubel, D. Novak, J., y Hanesian, H. (1978). Educational Psychology: A Cognitive View (2nd Ed.). New York: Holt, Rinehart \& Winston.

Delval, J. (1997). Tesis sobre el constructivismo. En Rodrigo, M.J. y Arnay J., La construcción del conocimiento escolar (pp. 15-35). Barcelona: Paidós.

Gómez Rijo, A. (2010). El alumnado como constructor de su propio aprendizaje en el área de educación física. Wanceulen E.F. Digital, 52-61, 6.

Bain, K. (2006). Lo que hacen los mejores profesores universitarios, PUV, Valencia.

Jornadas de Formación e Innovación Docente del Profesorado | № 1 (2018) Esta obra se distribuye con la licencia Creative Commons 